\title{
Morphological characterization and selection of castor bean accessions for mechanized production ${ }^{1}$
}

\author{
Sebastião Soares de Oliveira Neto², Matheus Kainan de Paula Manjavachi², \\ Douglas Mariani Zeffa ${ }^{3}$, Maria Márcia Pereira Sartori², Maurício Dutra Zanotto ${ }^{2}$
}

\section{ABSTRACT}

Castor bean (Ricinus communis L.) is an oilseed found in different regions worldwide, due to its easy propagation and adaptability. Cropping more productive disease-resistant genotypes that enable a mechanized production ensures greater economic returns for farmers. This study aimed to morphologically characterize and select promising castor bean accessions for mechanized cropping, mainly as a source of genetic variability for breeding programs with this purpose. Fifty accessions were assessed. Analysis of variance, dissimilarity clustering via the unweighted pair-group method with arithmetic mean (UPGMA) and principal component analysis were used to analyze the collected data. The dissimilarity analysis based on the Gower distance for qualitative and quantitative variables revealed three groups of accessions and the principal component analysis enabled the selection of those with desirable traits. The accessions BOC1, PRAT1 and SM2 exhibited morphoagronomic characteristics of interest to the mechanized production, such as ideal plant height, diameter, seed weight and oil content. Such genotypes show a potential for use as genitors in genetic breeding programs of castor bean.

KEYWORDS: Ricinus communis, mechanized agriculture, genetic breeding of oilseeds.

\section{INTRODUCTION}

The increase of gasoline prices has also increased the number of countries using renewable energy (Amigun et al. 2011), with Brazil and the United States as the largest producers and consumers of biodiesel (Brasil 2016). In the first five months of 2019 alone, Brazil produced 2.24 million $\mathrm{m}^{3}$ of biodiesel from different animal and plant sources (ANP 2019). Species not used in the human diet

\section{RESUMO}

Caracterização morfológica e seleção de acessos de mamoneira visando ao cultivo mecanizado

A mamoneira (Ricinus communis L.) é uma oleaginosa encontrada em várias regiões do mundo, devido à sua fácil propagação e adaptação. O cultivo de genótipos mais produtivos, resistentes a doenças e que permitem o uso de mecanização traz maior retorno econômico para os produtores. Objetivou-se realizar a caracterização morfológica e seleção de acessos de mamoneira promissores para o cultivo mecanizado, sobretudo como fonte de variabilidade para programas de melhoramento com este propósito. Cinquenta acessos foram avaliados. Os dados foram submetidos a análise de variância, análise de agrupamento pelo método UPGMA e análise de componentes principais. A análise de dissimilaridade baseada na distância de Gower para variáveis qualitativas e quantitativas revelou três grupos de acessos e a análise de componentes principais possibilitou a seleção daqueles com características desejáveis. Os acessos BOC1, PRAT1 e SM2 exibiram características morfoagronômicas de interesse para o cultivo mecanizado, tais como altura de planta, diâmetro, peso de sementes e teor de óleo ideais. Tais genótipos mostram potencial para uso como genitores em programas de melhoramento genético de mamoneira.

PALAVRAS-CHAVE: Ricinus communis, mecanização agrícola, melhoramento genético de oleaginosas.

show a potential as biodiesel sources, including castor bean (Ricinus communis L.) (Pecina-Quintero et al. 2013). In addition to its application in biodiesel, the castor oil, a product of castor beans, may be used in pharmaceutical products, cosmetics, soap, paint, plastics and lubricants (Ogunniyi 2006, Severino et al. 2012, Singh et al. 2015).

Because of its easy propagation, castor bean can be found in every continent, concentrated in tropical and subtropical regions (Govaerts et al. 2000,

1. Received: Jan. 21, 2019. Accepted: June 26, 2019. Published: Oct. 21, 2019. DOI: 10.1590/1983-40632019v4956749.

2. Universidade Estadual Paulista, Faculdade de Ciências Agronômicas, Departamento de Produção e Melhoramento Vegetal, Botucatu, SP, Brasil.E-mail/ORCID: neto.soliver@gmail.com/0000-0002-6372-4052, cid_agro@hotmail.com/ 0000-0002-7928-1540,mmpsartori@fca.unesp.br/0000-0003-4119-8642, zanotto@fca.unesp.br/0000-0003-3591-4788.

3. Universidade Estadual de Maringá, Departamento de Agronomia, Maringá, PR, Brasil.

E-mail/ORCID: douglas.mz@hotmail.com/0000-0002-0808-7230. 
Lakhani et al. 2015). Brazil is the third largest world producer (FAO 2018), with an increasing production, since the crop has been used in rotation after the soybean harvest in the Brazilian Savannah.

Plant breeding aimed at obtaining new castor bean cultivars is vital to the economic success of the crop, and planting more productive disease-resistant genotypes that allow the use of machinery contributes to achieve this goal.

For the mechanized harvesting of cultivars to be feasible, it is important to use suitable machinery (Rangel et al. 2003). Variability is also important in castor bean breeding programs, to incorporate commercially significant traits in new plants (Tan et al. 2012, Silva et al. 2019). Native or spontaneous germplasm collections are the primary source of this variability (Ellegren \& Galtier 2016).

Characterizing genotypes is the first step in defining the strategy for a plant breeding program (Moshkin 1986, Vieira et al. 2013, Varga et al. 2017, Silva et al. 2019). Morphological and agronomic descriptions of accessions are important for genotype assessment and characterization (Fufa et al. 2005, Silva et al. 2019), because they enable a commercial classification (UPOV 2014) and identify materials with desirable characteristics (Govindaraj et al. 2015).

This study aimed to morphologically characterize a collection of castor bean accessions from different regions of Brazil, and select those with suitable traits for use as a source of favorable alleles in breeding programs targeting mechanized cropping.

\section{MATERIAL AND METHODS}

Fifty castor bean accessions from three Brazilian states (Rio Grande do Norte, Minas Gerais and São Paulo) were assessed, all belonging to the germplasm bank of the Universidade Estadual Paulista: ATB1, ATB2, ATB3, BB1, BC1, BOC1, BOF1, BOF3, BOIT1, BTC1, BTC2, BTC3, BTC4, BTC5, BTC6, BTC7, BP1, CJ1, CJ2, CJ3, CJ4, CJ5, CJ6, CL1, IPE1, ML1, PARD1, PARD2, PRAT1, SI1, SAP1, SAP2, SAP3, SAP4, SBS1, SBS2, SBS3, SBS4, SJC1, SJC2, SM1 and SM2 were collected in São Paulo; CAMB1, CBJ1, GON1, GON2, PAR1 and PAR2 in Minas Gerais; and NAT1, NAT2 and NAT3 in Rio Grande do Norte.

The experiment was carried out in a greenhouse covered with polyethylene film and anti-insect screens on the sides, located in Botucatu, São Paulo

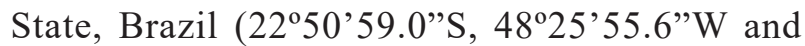
altitude of $786 \mathrm{~m}$ ). The average annual temperature and rainfall in the area are $21{ }^{\circ} \mathrm{C}$ and $1,528 \mathrm{~mm}$, respectively, and the climate is classified as Cwa, according to the Köppen's classification (warm temperate climate - mesothermal, with a wet summer and dry winter). The soil in the region is classified as Dystrophic Red Latosol (Embrapa 2006).

A completely randomized design with three repetitions was used. Seeds of the fifty treatments were planted directly into the soil on April 8, 2016. Approximately $200 \mathrm{~kg} \mathrm{ha}^{-1}$ of base dressing $\left(\mathrm{N}^{-} \mathrm{P}_{2} \mathrm{O}_{5}-\right.$ $\mathrm{K}_{2} \mathrm{O}$ ), in an 8-20-20 formulation, were applied. Each plot consisted of a furrow, with three seeds distributed in each. Plants were spaced $0.5 \mathrm{~m}$ apart, with $1.0 \mathrm{~m}$ between rows, and thinned when they were around $20 \mathrm{~cm}$ tall, leaving one plant per plot. The small plot size was due to the few seeds per accession. Drip irrigation was performed throughout the growth cycle, according to the water needs of the crop.

The castor bean accessions were morphologically characterized using 30 descriptors proposed by Brasil (2008), 7 quantitative and 23 qualitative. The quantitative descriptors were: plant height, primary raceme insertion height, stem diameter, number of internodes, number of commercial racemes, length of the primary raceme and 100 -seed weight. The seed oil content was determined by time-domain nuclear magnetic resonance (TD-NMR), using a spectrophotometer (SLK-SG-200, Spinlock Magnetic Resonance Solutions ${ }^{\circledR}$ ).

Data on qualitative stem traits were obtained by observing each plot. The descriptors considered were: stem wax, stem color and growth cycle. The leaf descriptors were: limb shape, vein color, limb wax and limb color, assessed in three leaves per plant, totaling nine leaves per accession.

To characterize the inflorescences, the following descriptors were observed in one raceme per plant: male flowers on the raceme, predominance of male flowers on the raceme, raceme density and raceme shape. The fruit descriptors assessed were: stigma color before pollination, fruit wax, fruit color, presence of spikes, spike density, spike color and fruit dehiscence in ten fruits per plant, totaling thirty fruits per accession.

The qualitative descriptors for seeds were evaluated in 100-seeds per accession for primary seed color, presence of secondary color, secondary color, 
type of secondary color, seed shape and caruncle protuberance.

The seed oil content was determined by timedomain nuclear magnetic resonance (TD-NMR), using a spectrophotometer (SLK-SG-200, Spinlock Magnetic Resonance Solutions ${ }^{\mathbb{R}}$ ).

The morphological descriptors were assessed in accordance with recommendations and at the relevant times stipulated by guidelines for identification, homogeneity and stability testing in castor bean plants (Brasil 2008). The data for these traits were classified into multiple categories (Table 1).

Analyses of variance (Anova) were applied to the quantitative data and, when the treatment effects were significant $(\mathrm{p}<0.05)$, their means were grouped by the Scott-Knott (1974) method. The accessions were hierarchically clustered using the UPGMA (unweighted pair-group method with arithmetic mean) method, based on the Gower distances (1971). The Mojena's method (1977) was applied to determine the cutoff point for the dendrogram, considering $\mathrm{k}=1.25$ as a stopping rule to define the number of groups (Milligan \& Cooper 1985). Clustering was validated by the cophenetic correlation coefficient (Sokal \& Rohlf 1962) and its significance calculated using the method described by Mantel (1967). A principal component analysis was carried out on the matrix data for accessions and traits (plant height, stem diameter, number of commercial racemes, length of the primary raceme, 100 -seed weight and seed oil content). These analyses were performed using the Minitab $17^{\circledR}$ (Minitab 2015) and R softwares (R Core Team 2019).

\section{RESULTS AND DISCUSSION}

The analysis of variance indicated significant $(p<0.05)$ inter-accession differences for all the studied quantitative traits (Table 2). The coefficient

Table 1. Morphological descriptors and phenological classes used to characterize the castor bean accessions.

\begin{tabular}{|c|c|}
\hline Descriptors & Phenological classes \\
\hline Stem wax & (1) Absent; (2) present. \\
\hline Stem color & $\begin{array}{l}\text { (1) Light green; (2) medium green; (3) dark green; (4) pinkish green; (5) pink; (6) red; } \\
\text { (7) reddish brown; (8) purple. }\end{array}$ \\
\hline Growth cycle & (1) Early: up to 30 days; (2) medium: 31 to 60 days; (3) late: over 60 days. \\
\hline Limb shape & (1) Flat; (2) slightly tapered; (3) tapered. \\
\hline Vein color & (1) Green; (2) red. \\
\hline Limb wax & (1) Absent; (2) present. \\
\hline Limb color & $\begin{array}{l}\text { (1) Light green; (2) green; (3) dark green; (4) pink; (5) reddish green; (6) red; } \\
\text { (7) purple. }\end{array}$ \\
\hline Male flowers on the raceme & (1) Absent; (2) present. \\
\hline Predominance of male flowers on the raceme & (1) Lower portion of the raceme; (2) interspersed among female flowers. \\
\hline Raceme density & (1) Sparse; (2) intermediate; (3) compact. \\
\hline Raceme shape & (1) Flask-shaped; (2) cylindrical; (3) conical. \\
\hline Stigma color before pollination & (1) Yellow; (2) green; (3) orange; (4) red; (5) pink. \\
\hline Fruit wax & (1) Absent; (2) present. \\
\hline Fruit color & $\begin{array}{l}\text { (1) Yellow; (2) light green; (3) medium green; (4) dark green; (5) pinkish green; } \\
\text { (6) pink; (7) red; (8) purple. }\end{array}$ \\
\hline Presence of spikes & (1) Absent; (2) present. \\
\hline Spike density & (1) Low; (2) medium; (3) high. \\
\hline Spike color & $\begin{array}{l}\text { (1) Yellow; (2) light green; (3) medium green; (4) dark green; (5) pinkish green; } \\
\text { (6) pink; (7) red; (8) purple. }\end{array}$ \\
\hline Fruit dehiscence & (1) Dehiscent; (2) semidehiscent; (3) indehiscent. \\
\hline Primary seed color & $\begin{array}{l}\text { (1) White; (2) yellow; (3) red; (4) light brown; (5) medium brown; (6) dark brown; } \\
\text { (7) reddish brown; (8) grey; (9) black. }\end{array}$ \\
\hline Presence of secondary color & (1) Absent; (2) present. \\
\hline Secondary color & $\begin{array}{l}\text { (1) White; (2) yellow; (3) red; (4) light brown; (5) medium brown; (6) dark brown; } \\
\text { (7) reddish brown; (8) grey; (9) black. }\end{array}$ \\
\hline Type of secondary color & (1) Speckled; (2) spotted, blotchy; (3) streaked. \\
\hline Seed shape & (1) Rounded; (2) ellipsoid. \\
\hline Caruncle protuberance & (1) Mild; (2) accentuated. \\
\hline
\end{tabular}


Table 2. Analyses of variance and coefficients of variation for quantitative traits measured in a collection of castor bean accessions.

\begin{tabular}{|c|c|c|c|c|c|c|c|c|c|}
\hline \multirow{2}{*}{ Source of variation } & \multirow{2}{*}{$\mathrm{DF}^{1}$} & \multicolumn{8}{|c|}{ Mean squares ${ }^{2}$} \\
\hline & & $\mathrm{PH}$ & RI & $\mathrm{S} \varnothing$ & NI & NR & RL & $100 \mathrm{SW}$ & $\% \mathrm{O}$ \\
\hline Accessions & 49 & $4,529.13 *$ & $3,189.88^{*}$ & $0.72 *$ & $14.97 *$ & $15.86^{*}$ & $144.77 *$ & $125.87 *$ & $46.85^{*}$ \\
\hline Error & 100 & 166.65 & 243.43 & 0.15 & 2.68 & 0.56 & 14.59 & 0.22 & 0.15 \\
\hline CV (\%) & - & 9.44 & 13.97 & 17.29 & 10.09 & 17.76 & 10.54 & 2.45 & 0.88 \\
\hline
\end{tabular}

${ }^{1} \mathrm{DF}$ : degrees of freedom. ${ }^{2} \mathrm{PH}$ : plant height $(\mathrm{cm})$; RI: primary raceme insertion height (cm); SØ: stem diameter (cm); NI: number of internodes; NR: number of commercial racemes; RL: raceme length (cm); 100SW: 100 -seed weight $(\mathrm{g}) ; \% \mathrm{O}$ : seed oil content $(\%)$. * Significant values at $5 \%$ of probability by the F-test.

of variation ranged from $0.88 \%$ (seed oil content) to $17.76 \%$ (number of commercial racemes), indicating a good experimental quality. The means of each accession for all these traits, with the respective groups identified according the Scott-Knott method, are shown in Table 3.

The plant height varied from $66.0 \mathrm{~cm}$ to $248.7 \mathrm{~cm}$ (Table 3), corroborating the results of Silva et al. (2017), who evaluated the genetic diversity of 208 castor bean lines and parental strains based on morpho-agronomic descriptors. They found plant heights that ranged between $45.0 \mathrm{~cm}$ and $189.1 \mathrm{~cm}$.

Although tall plants are not an obstacle to conventional farming techniques, small plants are important to facilitate mechanized cropping and harvesting operations (Lopes et al. 2008, Ferreira et al. 2009). The accessions BTC4 $(64.3 \mathrm{~cm}), \mathrm{CJ} 1$ $(66.7 \mathrm{~cm}), \mathrm{SJC} 1(69.3 \mathrm{~cm})$ and SJC2 $(74.7 \mathrm{~cm})$ showed the lowest values for this trait.

Another interesting characteristic for mechanized harvesting in castor bean is a low height of primary raceme insertion. The lowest means for this trait were recorded for the accessions BOF1 $(69.7 \mathrm{~cm})$, BTC4 $(53.0 \mathrm{~cm})$, CJ1 $(56.3 \mathrm{~cm})$, PRAT1 $(54.3 \mathrm{~cm}), \mathrm{SBS} 3(69.7 \mathrm{~cm}), \mathrm{SJC} 1(41.0 \mathrm{~cm})$ and SJC2 $(69.3 \mathrm{~cm})$. The highest values were for BTC5 $(181.3 \mathrm{~cm})$ and BTC6 $(170.7 \mathrm{~cm})$, what could compromise their mechanized harvest.

Small stem diameters also favor the mechanized harvest in castor bean, because they are easier for harvesters to cut. Despite the difference among the means, most the accessions were classified as having thin stems (less than $3.0 \mathrm{~cm}$ ), except for BB1 $(3.32 \mathrm{~cm}), \mathrm{BC} 1(3.28 \mathrm{~cm})$ and BTC6 $(3.72 \mathrm{~cm})$, with medium stem diameter values. According to Lopes et al. (2008) and Ferreira et al. (2009), thin stems are a desirable trait in castor bean plants.

In general, the number of internodes is directly related to plant height, that is, the lower the number of internodes, the smaller the plant height. Half of the analyzed accessions exhibited low number of internodes values, with the lowest obtained by ATB1 and SJC1 (11.66 and 12.33, respectively).

The number of commercial racemes is another important trait, because it contributes to the crop yield (Nóbrega et al. 2001). The highest values were recorded for the accessions BC1 (10.33), BTC5 (9.67), ATB3 (7.66), BTC3 (8.33), BTC6 and NAT3 (both 8.66). A large raceme length directly influences the number of castor bean fruits, which also affects the crop yield (Nobre et al. 2012). The accession with the longest raceme was BTC6 $(56.56 \mathrm{~cm})$.

Mean 100-seed weight values varied significantly between accessions, from $10.95 \mathrm{~g}$ (GON1) to $41.28 \mathrm{~g}$ (PRAT1). A high seed weight enables high grain yields (Savy Filho et al. 1999, Freire et al. 2007), with the most prominent accessions in the present study being PRAT1 (41.28 g), BOC1 (35.53 g), SM2 (31.75 g) and SM1 (28.71 g).

The seed oil content is an important trait, as well as one of the criteria used to select superior castor bean genotypes (Zimmerman 1958, Savy Filho et al. 1999, Freire et al. 2007, Milani et al. 2009). The means of the accessions studied here ranged between $32.0 \%$ and $50.8 \%$, with the highest ones for BTC2, BOC1, PRAT1, SAP3 and SM2 (all above $50 \%$ ).

The clustering of accessions by the UPGMA hierarchical method showed a high cophenetic correlation (0.89), considered significant based on the Mantel test $(\mathrm{p}<0.001)$. According to Sokal \& Rohlf (1962), cophenetic coefficients greater than 0.8 are desirable, because they indicate a good fit between clustering and distance matrices. A cutoff point at the distance of 0.32 in the obtained dendrogram, according to the Mojena's method (1977), allowed the identification of three distinct accession groups (Figure 1): group I (with black lines in the figure) consisted of 46 accessions, which exhibited a substantial genetic variability in the quantitative and qualitative analyzed traits; group II (green lines) contained accessions BOC1 
Table 3. Means for the quantitative traits ${ }^{1,2}$ measured in a collection of tested castor bean accessions.

\begin{tabular}{|c|c|c|c|c|c|c|c|c|}
\hline Accession & $\mathrm{PH}(\mathrm{cm})$ & RI (cm) & $\mathrm{S} \varnothing(\mathrm{cm})$ & NI & NR & $\mathrm{RL}(\mathrm{cm})$ & 100SW (g) & $\% \mathrm{O}$ \\
\hline ATB1 & $137.33 \mathrm{~d}$ & $115.33 \mathrm{c}$ & $2.17 \mathrm{c}$ & $14.00 \mathrm{c}$ & $4.66 \mathrm{~d}$ & $35.36 \mathrm{c}$ & $15.14 \mathrm{~m}$ & $35.20 \mathrm{~m}$ \\
\hline ATB2 & $115.00 \mathrm{f}$ & $85.00 \mathrm{~d}$ & $2.10 \mathrm{c}$ & $11.66 \mathrm{c}$ & $5.66 \mathrm{c}$ & $37.76 \mathrm{c}$ & $12.61 \mathrm{o}$ & 38.291 \\
\hline ATB3 & $209.33 \mathrm{~b}$ & $148.00 \mathrm{~b}$ & $2.97 \mathrm{~b}$ & $17.33 \mathrm{~b}$ & $7.66 \mathrm{~b}$ & $20.36 \mathrm{e}$ & $11.28 \mathrm{p}$ & $41.50 \mathrm{i}$ \\
\hline BB1 & $184.66 \mathrm{c}$ & $145.33 \mathrm{~b}$ & $3.32 \mathrm{a}$ & $17.00 \mathrm{~b}$ & $6.66 \mathrm{c}$ & $38.46 \mathrm{c}$ & 16.921 & $45.45 \mathrm{e}$ \\
\hline $\mathrm{BC} 1$ & $181.00 \mathrm{c}$ & $155.33 \mathrm{~b}$ & $3.28 \mathrm{a}$ & $19.00 \mathrm{~b}$ & $10.33 \mathrm{a}$ & $36.70 \mathrm{c}$ & $12.81 \mathrm{o}$ & $46.53 \mathrm{~d}$ \\
\hline BOC1 & $155.33 \mathrm{~d}$ & $141.33 \mathrm{~b}$ & $2.47 \mathrm{~b}$ & $17.00 \mathrm{~b}$ & $6.33 \mathrm{c}$ & $42.30 \mathrm{~b}$ & $35.53 \mathrm{~b}$ & $50.20 \mathrm{a}$ \\
\hline BOF1 & $76.66 \mathrm{~h}$ & $69.66 \mathrm{e}$ & $1.63 \mathrm{~d}$ & $14.66 \mathrm{c}$ & $2.66 \mathrm{e}$ & $26.93 \mathrm{~d}$ & $10.96 \mathrm{p}$ & $43.80 \mathrm{~g}$ \\
\hline BOF3 & $130.33 \mathrm{e}$ & $111.66 \mathrm{c}$ & $2.36 \mathrm{c}$ & $17.00 \mathrm{~b}$ & $2.66 \mathrm{e}$ & $35.43 \mathrm{c}$ & 17.641 & $42.30 \mathrm{~h}$ \\
\hline BOIT1 & $151.00 \mathrm{~d}$ & $128.33 \mathrm{c}$ & $2.08 \mathrm{c}$ & $17.33 \mathrm{~b}$ & $2.00 \mathrm{e}$ & $36.13 \mathrm{c}$ & $19.48 \mathrm{j}$ & $40.23 \mathrm{j}$ \\
\hline BP1 & $170.33 \mathrm{c}$ & $136.00 \mathrm{c}$ & $2.82 \mathrm{~b}$ & $17.66 \mathrm{~b}$ & $5.66 \mathrm{c}$ & $38.33 \mathrm{c}$ & $11.09 \mathrm{p}$ & $39.26 \mathrm{k}$ \\
\hline BTC1 & $141.66 \mathrm{~d}$ & $134.33 \mathrm{c}$ & $2.20 \mathrm{c}$ & $17.33 \mathrm{~b}$ & $3.00 \mathrm{e}$ & $28.46 \mathrm{~d}$ & $21.00 \mathrm{~h}$ & $46.53 \mathrm{~d}$ \\
\hline BTC2 & $132.00 \mathrm{e}$ & $117.66 \mathrm{c}$ & $1.74 \mathrm{~d}$ & $16.00 \mathrm{c}$ & $2.66 \mathrm{e}$ & $28.60 \mathrm{~d}$ & $19.39 \mathrm{j}$ & $50.80 \mathrm{a}$ \\
\hline BTC3 & $110.33 \mathrm{f}$ & $95.00 \mathrm{~d}$ & $2.42 \mathrm{~b}$ & $17.00 \mathrm{~b}$ & $8.33 \mathrm{~b}$ & $36.20 \mathrm{c}$ & $20.95 \mathrm{~h}$ & $41.86 \mathrm{i}$ \\
\hline BTC4 & $64.33 \mathrm{~h}$ & $53.00 \mathrm{e}$ & $1.63 \mathrm{~d}$ & $15.00 \mathrm{c}$ & $2.66 \mathrm{e}$ & $28.10 \mathrm{~d}$ & $23.87 \mathrm{f}$ & $45.86 \mathrm{e}$ \\
\hline BTC5 & $206.66 \mathrm{~b}$ & $181.33 \mathrm{a}$ & $2.91 \mathrm{~b}$ & $18.00 \mathrm{~b}$ & $9.67 \mathrm{a}$ & $37.60 \mathrm{c}$ & $24.47 \mathrm{f}$ & $47.90 \mathrm{~b}$ \\
\hline BTC6 & $248.67 \mathrm{a}$ & $170.66 \mathrm{a}$ & $3.72 \mathrm{a}$ & $16.66 \mathrm{~b}$ & $8.66 \mathrm{~b}$ & $56.56 \mathrm{a}$ & $27.04 \mathrm{e}$ & $46.30 \mathrm{~d}$ \\
\hline BTC7 & $150.33 \mathrm{~d}$ & $110.00 \mathrm{c}$ & $2.48 \mathrm{~b}$ & $16.33 \mathrm{~b}$ & $6.33 \mathrm{c}$ & $37.56 \mathrm{c}$ & $22.39 \mathrm{~g}$ & $40.06 \mathrm{j}$ \\
\hline CAMB1 & $115.00 \mathrm{f}$ & $102.00 \mathrm{~d}$ & $2.77 \mathrm{~b}$ & $17.00 \mathrm{~b}$ & $2.00 \mathrm{e}$ & $40.90 \mathrm{~b}$ & 17.481 & $45.13 \mathrm{e}$ \\
\hline CBJ1 & $162.66 \mathrm{c}$ & $139.00 \mathrm{~b}$ & $2.68 \mathrm{~b}$ & $15.00 \mathrm{c}$ & $5.33 \mathrm{~d}$ & $41.26 \mathrm{~b}$ & $22.02 \mathrm{~g}$ & $45.47 \mathrm{e}$ \\
\hline CJ1 & $66.66 \mathrm{~h}$ & $56.33 \mathrm{e}$ & $1.63 \mathrm{~d}$ & $14.66 \mathrm{c}$ & $2.33 \mathrm{e}$ & $33.53 \mathrm{c}$ & $18.25 \mathrm{k}$ & $47.77 \mathrm{~b}$ \\
\hline CJ2 & $95.00 \mathrm{~g}$ & $75.33 \mathrm{~d}$ & $1.71 \mathrm{~d}$ & $14.66 \mathrm{c}$ & $2.66 \mathrm{e}$ & $37.90 \mathrm{c}$ & $24.06 \mathrm{f}$ & $39.67 \mathrm{k}$ \\
\hline $\mathrm{CJ} 3$ & $143.00 \mathrm{~d}$ & $125.66 \mathrm{c}$ & $2.26 \mathrm{c}$ & $15.33 \mathrm{c}$ & $2.00 \mathrm{e}$ & $36.30 \mathrm{c}$ & $24.07 \mathrm{f}$ & $46.10 \mathrm{~d}$ \\
\hline CJ4 & $168.33 \mathrm{c}$ & $132.67 \mathrm{c}$ & $2.35 \mathrm{c}$ & $17.00 \mathrm{~b}$ & $2.33 \mathrm{e}$ & $43.50 \mathrm{~b}$ & $24.69 \mathrm{f}$ & $45.77 \mathrm{e}$ \\
\hline CJ5 & $144.00 \mathrm{~d}$ & $124.33 \mathrm{c}$ & $2.32 \mathrm{c}$ & $16.00 \mathrm{c}$ & $1.66 \mathrm{e}$ & $40.63 \mathrm{~b}$ & $14.33 \mathrm{n}$ & $43.90 \mathrm{~g}$ \\
\hline CJ6 & $146.33 \mathrm{~d}$ & $132.33 \mathrm{c}$ & $1.99 \mathrm{c}$ & $14.66 \mathrm{c}$ & $4.33 \mathrm{e}$ & $40.80 \mathrm{~b}$ & $22.90 \mathrm{~g}$ & $45.30 \mathrm{e}$ \\
\hline GON1 & $108.33 \mathrm{f}$ & $100.66 \mathrm{~d}$ & $1.97 \mathrm{c}$ & $14.66 \mathrm{c}$ & $2.66 \mathrm{e}$ & $37.46 \mathrm{c}$ & $10.95 \mathrm{p}$ & $39.17 \mathrm{k}$ \\
\hline GON2 & $111.33 \mathrm{f}$ & $77.00 \mathrm{~d}$ & $2.79 \mathrm{~b}$ & $17.00 \mathrm{~b}$ & $5.00 \mathrm{~d}$ & $41.76 \mathrm{~b}$ & $12.47 \mathrm{o}$ & 38.331 \\
\hline IPE1 & $170.66 \mathrm{c}$ & $87.66 \mathrm{~d}$ & $2.12 \mathrm{c}$ & $16.00 \mathrm{c}$ & $5.00 \mathrm{~d}$ & $19.20 \mathrm{e}$ & $14.18 \mathrm{n}$ & $39.40 \mathrm{k}$ \\
\hline ML1 & $182.66 \mathrm{c}$ & $125.00 \mathrm{c}$ & $2.48 \mathrm{~b}$ & $17.00 \mathrm{~b}$ & $5.33 \mathrm{~d}$ & $38.36 \mathrm{c}$ & $20.05 \mathrm{i}$ & $44.10 \mathrm{f}$ \\
\hline NAT1 & $155.66 \mathrm{~d}$ & $126.33 \mathrm{c}$ & $2.14 \mathrm{c}$ & $15.00 \mathrm{c}$ & $4.66 \mathrm{~d}$ & $28.46 \mathrm{~d}$ & $14.24 \mathrm{n}$ & 38.631 \\
\hline NAT2 & $164.33 \mathrm{c}$ & $142.66 \mathrm{~b}$ & $2.48 \mathrm{~b}$ & $13.33 \mathrm{c}$ & $5.33 \mathrm{~d}$ & $33.50 \mathrm{c}$ & $11.61 \mathrm{p}$ & $41.47 \mathrm{i}$ \\
\hline NAT3 & $128.66 \mathrm{e}$ & $80.33 \mathrm{~d}$ & $2.28 \mathrm{c}$ & $15.33 \mathrm{c}$ & $8.66 \mathrm{~b}$ & $26.16 \mathrm{~d}$ & $12.89 \mathrm{o}$ & $42.23 \mathrm{~h}$ \\
\hline PAR1 & $100.66 \mathrm{f}$ & $85.00 \mathrm{~d}$ & $1.72 \mathrm{~d}$ & $14.00 \mathrm{c}$ & $2.66 \mathrm{e}$ & $27.90 \mathrm{~d}$ & $15.40 \mathrm{~m}$ & $44.30 \mathrm{f}$ \\
\hline PAR2 & $133.66 \mathrm{e}$ & $118.33 \mathrm{c}$ & $2.23 \mathrm{c}$ & $16.33 \mathrm{~b}$ & $4.66 \mathrm{~d}$ & $28.16 \mathrm{~d}$ & 16.751 & $45.60 \mathrm{e}$ \\
\hline PARD1 & $133.66 \mathrm{e}$ & $120.66 \mathrm{c}$ & $2.15 \mathrm{c}$ & $13.33 \mathrm{c}$ & $2.66 \mathrm{e}$ & $37.33 \mathrm{c}$ & $24.15 \mathrm{f}$ & $46.46 \mathrm{~d}$ \\
\hline PARD2 & $154.66 \mathrm{~d}$ & $143.33 \mathrm{~b}$ & $2.68 \mathrm{~b}$ & $16.00 \mathrm{c}$ & $2.66 \mathrm{e}$ & $45.60 \mathrm{~b}$ & $18.27 \mathrm{k}$ & $44.66 \mathrm{f}$ \\
\hline PRAT1 & $154.33 \mathrm{~d}$ & $54.33 \mathrm{e}$ & $2.73 \mathrm{~b}$ & $15.66 \mathrm{c}$ & $2.00 \mathrm{e}$ & $38.54 \mathrm{c}$ & $41.28 \mathrm{a}$ & $50.83 \mathrm{a}$ \\
\hline SAP1 & $125.00 \mathrm{e}$ & $116.67 \mathrm{c}$ & $1.95 \mathrm{c}$ & $19.00 \mathrm{~b}$ & $2.33 \mathrm{e}$ & $28.60 \mathrm{~d}$ & $15.17 \mathrm{~m}$ & $48.33 \mathrm{~b}$ \\
\hline SAP2 & $110.67 \mathrm{f}$ & $99.00 \mathrm{~d}$ & $2.44 \mathrm{~b}$ & $19.00 \mathrm{~b}$ & $6.00 \mathrm{c}$ & $38.70 \mathrm{c}$ & 17.321 & $47.40 \mathrm{~b}$ \\
\hline SAP3 & $178.66 \mathrm{c}$ & $151.67 \mathrm{~b}$ & $1.95 \mathrm{c}$ & $22.66 \mathrm{a}$ & $2.66 \mathrm{e}$ & $46.93 \mathrm{~b}$ & $20.92 \mathrm{~h}$ & $50.40 \mathrm{a}$ \\
\hline SAP4 & $145.67 \mathrm{~d}$ & $136.67 \mathrm{c}$ & $2.65 \mathrm{~b}$ & $19.00 \mathrm{~b}$ & $2.33 \mathrm{e}$ & $42.00 \mathrm{~b}$ & $20.07 \mathrm{i}$ & $45.50 \mathrm{e}$ \\
\hline SBS1 & $132.00 \mathrm{e}$ & $114.33 \mathrm{c}$ & $2.16 \mathrm{c}$ & $16.00 \mathrm{c}$ & $2.33 \mathrm{e}$ & $39.16 \mathrm{c}$ & $20.36 \mathrm{i}$ & $35.83 \mathrm{~m}$ \\
\hline SBS2 & $134.33 \mathrm{e}$ & $126.33 \mathrm{c}$ & $2.34 \mathrm{c}$ & $16.66 \mathrm{~b}$ & $2.33 \mathrm{e}$ & $38.10 \mathrm{c}$ & $22.65 \mathrm{~g}$ & $47.83 \mathrm{~b}$ \\
\hline SBS3 & $87.33 \mathrm{~g}$ & $69.66 \mathrm{e}$ & $1.84 \mathrm{~d}$ & $16.33 \mathrm{~b}$ & $5.66 \mathrm{c}$ & $43.63 \mathrm{~b}$ & $18.46 \mathrm{k}$ & $43.40 \mathrm{~g}$ \\
\hline SBS4 & $107.66 \mathrm{f}$ & $84.33 \mathrm{~d}$ & $1.46 \mathrm{~d}$ & $16.66 \mathrm{~b}$ & $2.33 \mathrm{e}$ & $43.30 \mathrm{~b}$ & $19.18 \mathrm{j}$ & $40.16 \mathrm{j}$ \\
\hline SI1 & $108.33 \mathrm{f}$ & $88.33 \mathrm{~d}$ & $2.05 \mathrm{c}$ & $13.00 \mathrm{c}$ & $2.66 \mathrm{e}$ & $43.36 \mathrm{~b}$ & $11.29 \mathrm{p}$ & $45.30 \mathrm{e}$ \\
\hline SJC1 & $69.33 \mathrm{~h}$ & $41.00 \mathrm{e}$ & $1.40 \mathrm{~d}$ & $12.33 \mathrm{c}$ & $5.66 \mathrm{c}$ & $35.36 \mathrm{c}$ & $13.02 \mathrm{o}$ & $39.57 \mathrm{k}$ \\
\hline SJC2 & $74.66 \mathrm{~h}$ & $69.33 \mathrm{e}$ & $1.68 \mathrm{~d}$ & $15.33 \mathrm{c}$ & $1.66 \mathrm{e}$ & $28.73 \mathrm{~d}$ & $12.14 \mathrm{o}$ & $45.57 \mathrm{e}$ \\
\hline SM1 & $89.33 \mathrm{~g}$ & $84.00 \mathrm{~d}$ & $1.75 \mathrm{~d}$ & $14.00 \mathrm{c}$ & $2.66 \mathrm{e}$ & $28.43 \mathrm{~d}$ & $28.71 \mathrm{~d}$ & $47.20 \mathrm{c}$ \\
\hline SM2 & $165.33 \mathrm{c}$ & $154.67 \mathrm{~b}$ & $2.21 \mathrm{c}$ & $24.00 \mathrm{a}$ & $2.33 \mathrm{e}$ & $37.60 \mathrm{c}$ & $31.75 \mathrm{c}$ & $50.77 \mathrm{a}$ \\
\hline
\end{tabular}

${ }^{1} \mathrm{PH}$ : plant height; RI: primary raceme insertion height; SØ: stem diameter; NI: number of internodes; NR: number of commercial racemes; RL: raceme length; 100SW: 100 -seed weight; $\% \mathrm{O}$ : seed oil content. ${ }^{2}$ Means followed by the same letter in the column belong to the same group, according to the Scott-Knott test at $5 \%$ of probability. 
and SM2, with elevated 100-seed weight and seed oil content; group III (blue lines) included the accessions BTC5 and BTC6, characterized as the plants with the highest means for height and primary raceme insertion.

In summary, the most desirable morphoagronomic characteristics for castor bean breeding programs targeting mechanized cropping are small plants with a high primary raceme insertion height, fine to medium stem diameter, large number of commercial racemes, superior seed weight, long racemes and high seed oil content. The multivariate analysis exploiting the inter-relations among traits (plant height, stem diameter, number of commercial racemes, length of the primary raceme, 100-seed weight and seed oil content) and accessions, by principal component analysis (Table 4, Figure 2), allowed to identify desirable combinations of accessions and phenotypic traits for this proposal of breeding programs.

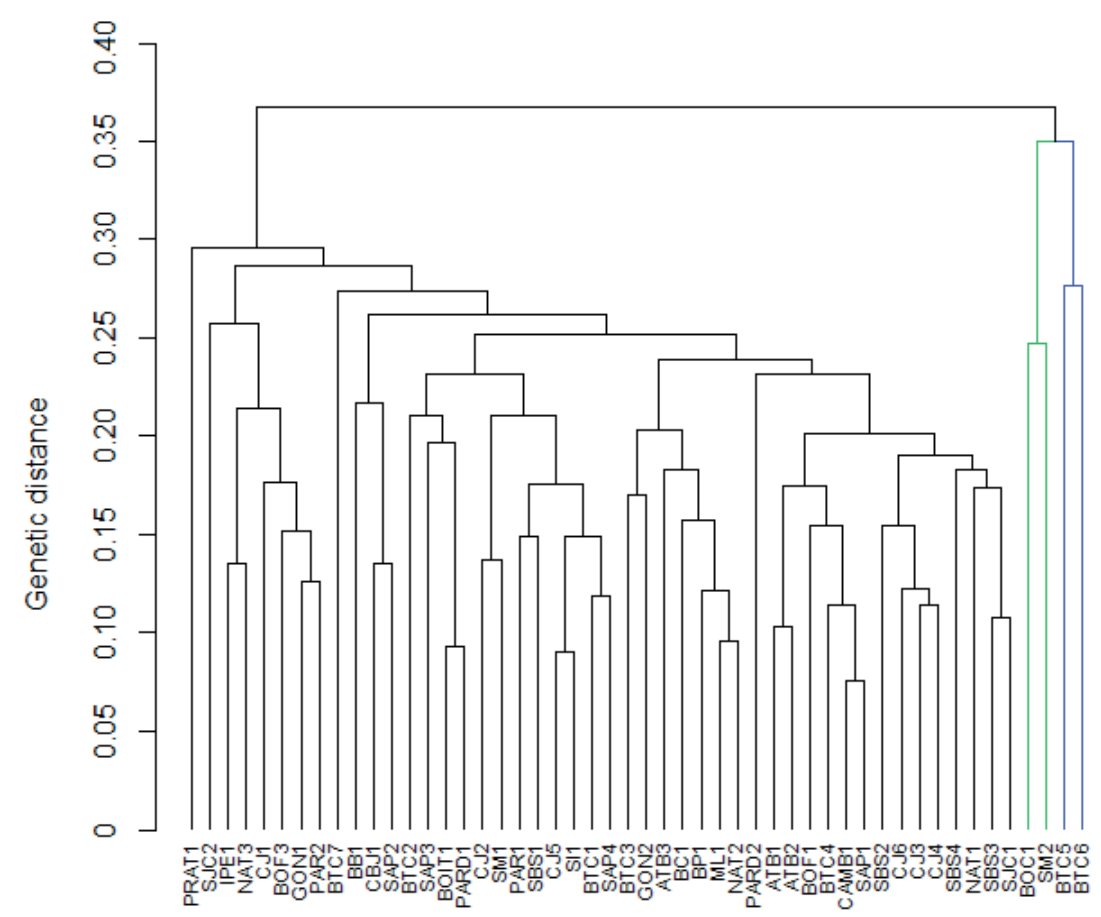

Figure 1. Dendrogram obtained via UPGMA method (with Gower distances) to cluster fifty Ricinus communis accessions characterized for morphological quantitative and qualitative traits $\left(r_{\text {cophenetic }}=0.89 ; \mathrm{p}<0.001\right)$.

Table 4. Statistics for a principal component analysis applied to data of six agronomic traits ${ }^{1}$ used to characterize a collection of fifty castor bean accessions.

\begin{tabular}{|c|c|c|c|c|c|c|c|}
\hline \multirow{2}{*}{\multicolumn{2}{|c|}{ Statistics }} & \multicolumn{6}{|c|}{ Principal components } \\
\hline & & $\mathrm{PC} 1$ & $\mathrm{PC} 2$ & $\mathrm{PC} 3$ & $\mathrm{PC} 4$ & PC5 & PC6 \\
\hline \multicolumn{2}{|c|}{ Eigenvalues } & 2.400 & 1.6711 & 0.855 & 0.466 & 0.420 & 0.186 \\
\hline \multicolumn{2}{|c|}{ Proportion } & 0.400 & 0.2780 & 0.143 & 0.078 & 0.070 & 0.031 \\
\hline \multicolumn{2}{|c|}{ Cumulative } & 0.400 & 0.6790 & 0.821 & 0.899 & 0.969 & 1.000 \\
\hline \multicolumn{8}{|c|}{ Correlation matrix } \\
\hline \multirow{6}{*}{ Trait } & PH & 0.561 & 0.104 & 0.122 & -0.533 & 0.210 & -0.576 \\
\hline & $\mathrm{S} \varnothing$ & 0.583 & 0.175 & 0.001 & -0.214 & -0.118 & 0.755 \\
\hline & NR & 0.380 & 0.443 & 0.264 & 0.743 & -0.023 & -0.190 \\
\hline & RL & 0.333 & -0.236 & -0.826 & 0.208 & -0.273 & -0.184 \\
\hline & $100 \mathrm{SW}$ & 0.240 & -0.610 & 0.080 & 0.270 & 0.687 & 0.141 \\
\hline & $\% \mathrm{O}$ & 0.181 & 0.181 & 0.477 & 0.039 & -0.629 & -0.093 \\
\hline
\end{tabular}

${ }^{1}$ PH: plant height; SØ: stem diameter; NR: number of racemes; RL: raceme length; 100SW: 100-seed weight; \%O: seed oil content. 


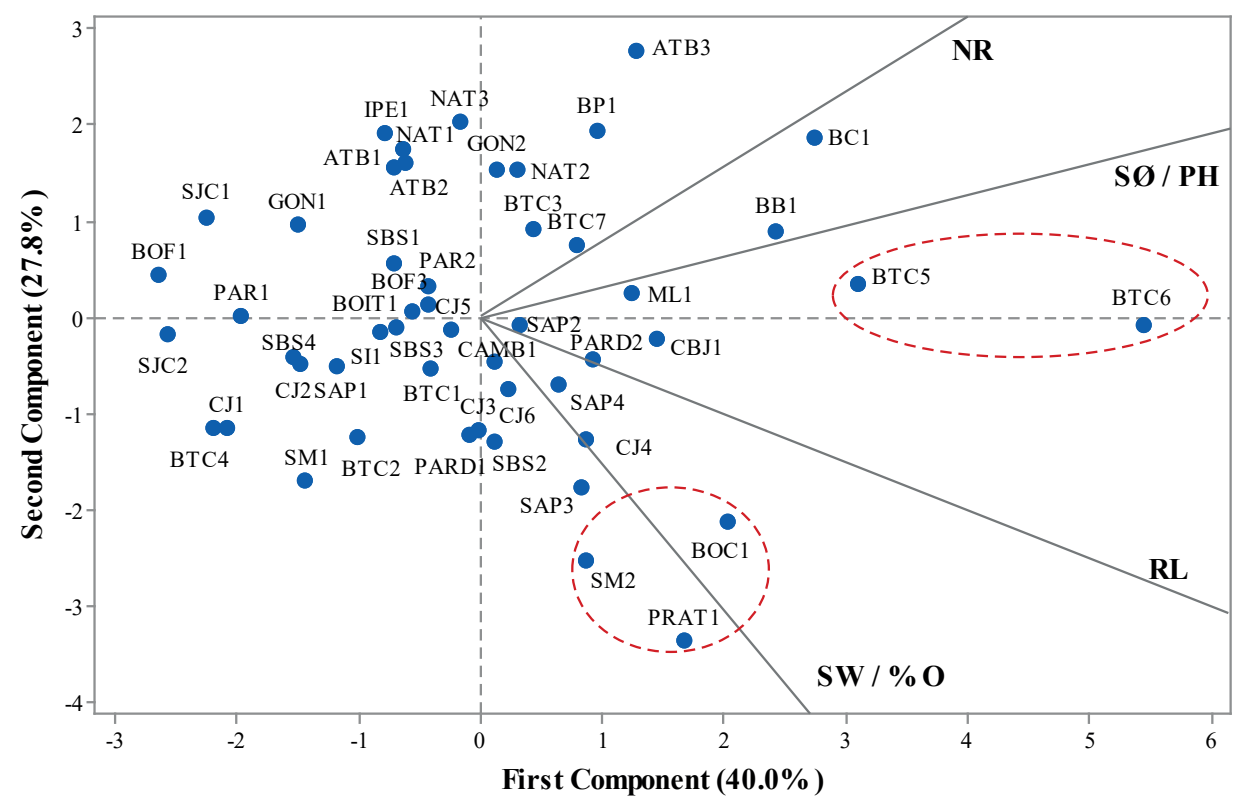

Figure 2. Biplot of principal component analysis for data of six agronomic traits (coded lines) used to characterize a collection of fifty castor bean accessions (blue points). PH: plant height; SØ: stem diameter; NR: number of racemes; RL: raceme length; SW: 100-seed weight; \%O: seed oil content.

From the fifty genotypes, five accessions (BTC5, BTC6, BOC1, PRAT1 and SM2), highlighted in Figure 2, were selected as potential sources of favorable alleles for castor bean breeding programs. These accessions were from different groups in the dissimilarity analysis (greater genetic distance between them) and, as such, they may be used in crossings with other commercial cultivars, in order to combine promising characteristics. SM2, BOC1 and PRAT1 stood out for displaying a combination of ideal characteristics: height between $154 \mathrm{~cm}$ and $165 \mathrm{~cm}$, stem diameter from $2.2 \mathrm{~cm}$ to $2.7 \mathrm{~cm}, 100$ seed weight between $31.7 \mathrm{~g}$ and $41.3 \mathrm{~g}$ and superior seed oil content (50.2\% to $50.8 \%$ ).

\section{CONCLUSIONS}

1. A significant genetic variability was observed among the fifty castor bean accessions. The accessions BTC5, BTC6, BOC1, PRAT1 and SM2 are the most divergent ones, and are recommended to be used in crossings to combine promising characteristics;

2. The accessions BOC1, PRAT1 and SM2 have morpho-agronomic characteristics of commercial interest, especially for mechanized harvesting, such as ideal plant height, diameter, seed weight and oil content. Therefore, they are promising genotypes as a source of favorable alleles in castor bean genetic breeding programs.

\section{REFERENCES}

AGÊNCIA NACIONAL DO PETRÓLEO, GÁS NATURAL E BIOCOMBUSTÍVEIS (ANP). Informações de mercado. 2019. Available at: http://www.anp.gov.br/ producao-de-biocombustiveis/biodiesel/informacoes-demercado. Access on: 27 June 2019.

AMigun, B.; MusAngO, J. K.; BREnT, A. C. Community perspectives on the introduction of biodiesel production in the Eastern Cape province of South Africa. Energy, v. 36, n. 5, p. 2502-2508, 2011.

BRASIL. Ministério da Agricultura, Pecuária e Abastecimento. Instruções para execução dos ensaios de distinguibilidade, homogeneidade e estabilidade de cultivares de mamona (Ricinus communis L.). 2008. Available at: http://www.agricultura.gov.br/assuntos/ insumos-agropecuarios/insumos-agricolas/protecao-decultivar/agricolas. Access on: 31 Aug. 2017.

BRASIL. Ministério de Minas e Energia. Boletim mensal dos biocombustiveis. 2016. Available at: http:// www.mme.gov.br/documents/1138769/1732805/Bolet $\mathrm{im}+\mathrm{DBio}+\mathrm{n} \% \mathrm{C} 2 \% \mathrm{BA}+106+$ dezembro+de+2016.pdf/ d0fd7bc5-b800-443e-a0f6-2959e7dd8a8e. Access on: 27 June 2019. 
ELLEGREN, H.; GALTIER, N. Determinants of genetic diversity. Nature, v. 17, n. 7, p. 422-433, 2016.

EMPRESA BRASILEIRA DE PESQUISA AGROPECUÁRIA (Embrapa). Sistema brasileiro de classificação de solos. Rio de Janeiro: Embrapa Solos, 2006.

FERREIRA, M. G. C.; MARUYAMA, W. I.; SORATTO, R. P. Avaliação de cultivares de mamona em dois arranjos de plantas no outono-inverno em Cassilândia - MS. Revista Brasileira de Oleaginosas e Fibrosas, v. 13, n. 2, p. 5360, 2009.

FOOD AND AGRICULTURE ORGANIZATION OF THE UNITED NATIONS (FAO). Food and agricultural commodities production. 2018. Available at: http://www. fao.org. Access on: 5 Apr. 2018.

FREIRE, R. M. M.; LIMA, E. F.; ANDRADE, F. P.; MILANI, M.; NÓBREGA, M. B. de M. Melhoramento genético. In: AZEVEDO, D. M. P.; BELTRÃO, N. E. de M. (ed.). O agronegócio da mamona no Brasil. Campina Grande: Embrapa Algodão, 2007. p. 169-194.

FUFA, H.; BAENZIGER, P. S.; BEECHER, B. S.; DWEIKAT, I.; GRAYBOSCH, R. A.; ESKRIDGE, K. $\mathrm{M}$. Comparison of phenotypic and molecular markerbased classifications of hard red winter wheat cultivars. Euphytica, v. 145, n. 1, p. 133-146, 2005.

GOVAERTS, R.; FRODIN, D. G.; RADCLIFFE-SMITH, A. World checklist and bibliography of Euphorbiaceae (and Pandaceae). Richmond: Royal Botanic Gardens Kew, 2000.

GOVINDARAJ, M.; VETRIVENTHAN, M.; SRINIVASAN, M. Importance of genetic diversity assessment in crop plants and its recent advances: an overview of its analytical perspectives. Genetics Research International, v. 1, n. 1, p. 1-14, 2015.

GOWER, J. C. A. A general coefficient of similarity and some of its properties. Biometrics, v. 27, n. 4, p. 857-874, 1971.

LAKHANI, H. N.; PATEL, S. V.; BODAR, N. P.; GOLAKIYA, B. A. RAPD analysis of genetic diversity of castor bean (Ricinus communis L.). International Journal of Current Microbiology and Applied Sciences, v. 4, n. 1, p. 696-703, 2015.

LOPES, F. F. de M.; BELTRÃO, N. E. de M.; LOPES NETO, J. P.; PEDROZA, J. P. Crescimento inicial de genótipos de mamoneira com sementes submetidas ao envelhecimento acelerado. Revista Brasileira de Oleaginosas e Fibrosas, v. 12, n. 2, p. 69-79, 2008.

MANTEL, N. The detection of disease clustering and a generalized regression approach. Cancer Research, v. 27, n. 1, p. 209-220, 1967.
MILANI, M.; NÓBREGA, M. B. M.; ANDRADE, F. P. Andamento e perspectivas do programa de melhoramento de mamona da Embrapa. Campina Grande: Embrapa Algodão, 2009.

MINITAB: statistical software data analysis software. 2015. Available at: https://www.minitab.com/pt-br/. Access on: 09 May 2019.

MOJENA, R. Hierarchical grouping methods and stopping rules: an evaluation. The Computer Journal, v. 20, n. 1, p. 359-363, 1977.

MOSHKIN, V. A. Castor. Moskow: Kolos, 1986.

NOBRE, R. G.; LIMA, G. S. de; GHEYI, H. R.; MEDEIROS, E. P. de; SOARES, L. A. A.; ALVES, A. $\mathrm{N}$. Teor de óleo e produtividade da mamoneira de acordo com a adubação nitrogenada e irrigação com água salina. Pesquisa Agropecuária Brasileira, v. 47, n. 7, p. 991-999, 2012.

NÓBREGA, M. B. M.; ANDRADE, F. P.; SANTOS, J. W.; MILANI, M.; LEITE, E. J. Germoplasma. In: AZEVEDO, D. M. P. de; LIMA, E. F. (ed.). O agronegócio da mamona no Brasil. Brasília, DF: Embrapa Informação Tecnológica, 2001. p. 257-280.

OGUNNIYI, D. S. Castor oil: a vital industrial raw material. Bioresource Technology, v. 97, n. 9, p. 10861091, 2006.

PECINA-QUINTERO, V.; ANAYA-LÓPEZ, J. L.; NÚÑEZ-COLÍN, C. A.; ZAMARRIPA-COLMENERO, A.; MONTES-GARCÍA, N.; SOLÍS-BONILLA, J. L.; AGUILAR-RANGEL, M. R. Assessing the genetic diversity of castor bean from Chiapas, México, using SSR and AFLP markers. Industrial Crops and Products, v. 41, n. 1, p. 134-143, 2013.

R CORE TEAM. $R$ : a language and environment for statistical computing. 2019. Available at: https://www.Rproject.org. Access on: 10 May 2019.

RANGEL, L. E. P.; FERREIRA, L. G.; ALMEIRA, V. M. de; MENEZES, V. L. Mamona: situação atual e perspectivas no Mato Grosso. Campina Grande: Embrapa Algodão, 2003. (Documentos, 106).

SAVY FILHO, A.; BANZATTO, N. V.; VEIGA, R. F. de A.; CHIAVEGATO, E. J.; CAMARGO, C. E. de O.; CAMPO-DALL'ORTO, F. A.; GODOY, I. J. de; FAZUOLI, L. C.; CARBONELL, S. A. M.; SIQUEIRA, W. J. Descritores mínimos para o registro institucional de cultivares: mamona. Campinas: Instituto Agronômico, 1999.

SCOTT, A. J.; KNOTT, M. A. Cluster analysis method for grouping means in the analysis of variance. Biometris, v. 30, n. 3 , p. 507-512, 1974. 
SEVERINO, L. S.; AULD, D. L.; BALDANZI, M.; CÂNDIDO, M. J. D.; CHEN, G.; CROSBY, W.; TAN, D.; HE, X.; LAKSHMAMMA, P.; LAVANYA, C.; MACHADO, O. L. T.; MIELKE, T.; MILLER, T. D.; MORRIS, J. B.; MORSE, S. A.; NAVAS, A. A.; SOARES, D. J.; SOFIATTI, V.; WANG, M. L.; ZANOTTO, M. D.; ZIELER, H. Review on the challenges for increased production of castor. Agronomy Journal, v. 104, n. 4, p. 853-880, 2012.

SILVA, A. R. S.; SILVA, S. A.; SANTOS, L. A. dos; SOUZA, D. R. de; ARAUJO, G. de M.; DANTAS, J. L. L.; LEITE, E. da S.; DANTAS, A. C. V. L. Characterization and performance of castor bean lineages and parents at the UFRB germplasm bank. Plos One, v. 14, n. 1, p. 1-15, 2019.

SILVA, A. R.; SILVA, S. A.; SANTOS, A. A. dos; SOUZA, D. R. de; ARAÚJO, G. de M.; MOREIRA, R. F. C. Genetic divergence among castor bean lines and parental strains using ward's method based on morphoagronomic descriptors. Acta Scientiarum, v. 39, n. 3, p. 307-313, 2017.

SINGH, A. S.; KUMARI, S.; ARPAN, R. M.; BHAVESH, B. G.; SUBHASH, N.; NITISH, K. Role of conventional and biotechnological approaches in genetic improvement of castor (Ricinus communis L.). Industrial Crops and Products, v. 74, n. 1, p. 55-62, 2015.
SOKAL, R. R.; ROHLF, F. J. The comparison of dendrograms by objective methods. Taxon, v. 11, n. 2, p. 33-40, 1962.

TAN, H.; MANMAN, T.; QIAN, L.; YONGPENG, Z.; JIA, L.; HUANXIU, L. A review of molecular makers applied in cowpea (Vigna unguiculata L.Walp.) breeding. Journal of Life Science, v. 6, n. 1, p. 1190-1199, 2012.

UPOV. International Union for the Protection of New Varieties of Plants. Guidelines for the conduct of tests for distinctness, uniformity and stability: castor bean. Geneva: UPOV, 2014.

VARGA, F.; CAROVIĆ-STANKO, K.; RISTIĆ, M.; GRDIŠA, M.; LIBER, Z.; ŠATOVIĆ, Z. Morphological and biochemical intraspecific characterization of Ocimum basilicum L. Industrial Crops and Products, v. 109, n. 1, p. 611-618, 2017.

VIEIRA, E. A.; FIALHO, J. de F.; FALEIRO, F. G.; BELLON, G.; FONSECA, K. G. da; SILVA, M. S.; PAULA-MORAES, S. V. de; CARVALHO, L. J. C. B. Caracterização fenotípica e molecular de acessos de mandioca de indústria com potencial de adaptação às condições do Cerrado do Brasil Central. Semina: Ciências Agrárias, v. 34, n. 2, p. 567-582, 2013.

ZIMMERMAN, L. H. Castor beans: a new oil crop for mechanized production. Advances in Agronomy, v. 10, n. 1, p. 257-288, 1958. 